Research Article

\title{
Particle Production at CBM Energies in a Thermal Model Approach
}

\author{
A. Prakash, P. K. Srivastava, and B. K. Singh \\ Department of Physics, Banaras Hindu University, Varanasi 221005, India \\ Correspondence should be addressed to P. K. Srivastava; prasul11@gmail.com
}

Received 25 November 2013; Accepted 16 January 2014; Published 4 March 2014

Academic Editor: Terry Sloan

Copyright (C) 2014 A. Prakash et al. This is an open access article distributed under the Creative Commons Attribution License, which permits unrestricted use, distribution, and reproduction in any medium, provided the original work is properly cited. The publication of this article was funded by $\mathrm{SCOAP}^{3}$.

\begin{abstract}
The compressed baryonic matter (CBM) experiment planned at the Facility for Antiproton and Ion Research (FAIR) will provide a major scientific effort for exploring the properties of strongly interacting matter in the high baryon density regime. One of the important goals behind such experiment is to precisely determine the equation of state (EOS) for the strongly interacting matter at extremely large baryon density. In this paper, we have used some successful models for RHIC and LHC energies to predict different particle ratios and the total multiplicity of various hadrons in the CBM energy range, that is, from $10 \mathrm{~A} \mathrm{GeV}$ to $40 \mathrm{~A} \mathrm{GeV}$ lab energies, which corresponds to $4.43 \mathrm{~A} \mathrm{GeV}$ and $8.71 \mathrm{~A} \mathrm{GeV}$ center-of-mass energies. Our main emphasis is to estimate the strange particles enhancement as well as an increase in the net baryon density at CBM experiment. We have also compared the model results with the experimental data obtained at alternating gradient synchrotron (AGS) and super proton synchrotron (SPS).
\end{abstract}

\section{Introduction}

Relativistic heavy ion collisions provide a unique way to create and investigate the QCD matter at extreme temperature $(T)$ and/or density $\left(\rho_{B}\right)[1,2]$. These experiments are expected to produce short lived bubbles of medium, in which the quarks and gluons are the active degrees of freedom [3]. However, the analysis of such collision events is complicated due to a transient nature of the reaction. In these reactions, the object of interest is the formation of a fireball which consists of a compressed and/or heated zone and it is of finite small size (order of few Fermi) and further it fades away in a very short duration of less than $10^{-22}$ seconds [4]. The only observation comes through the particles (most abundantly hadrons) produced and emitted from the collision zone into the detectors. The main features of particle multiplicity, that is, the variation of number of particles produced with respect to collision energy, the momentum spectra, and the relative abundances of different species, have led us in the understanding of the nature and properties of strong interactions $[5,6]$. Several experimental programs were planned to explore the properties of strongly interacting matter and to search the possible existence of QGP, for example, alternating gradient synchrotron (AGS) and relativistic heavy-ion collider (RHIC) experiments at BNL [7-9], super proton synchrotron (SPS) and large hadron collider (LHC) at CERN [10-12], and so forth. The compressed baryonic matter (CBM) experiment at Facility for Antiproton and Ion Research (FAIR) machine will provide a similar hot, dense situation in the laboratory. The aim of the CBM experiment is to obtain information on (i) the properties of hadrons in dense or hot baryonic/hadronic matter, (ii) the restoration of chiral symmetry at high temperatures and high baryon densities, (iii) the deconfinement phase transition from hadronic to quark-gluon matter at high temperatures and/or high baryon densities, and (iv) the nuclear equation of state at high baryon densities $[13,14]$.

These experimental efforts need reliable theoretical guidance to proceed in the right direction. Lattice QCD which is the first principle method to calculate the properties of QCD provides some quality information on the strongly interacting matter at finite temperature and zero (or very 
small) chemical potential $(\mu)$ [15-17]. However, lattice QCD approach is severely limited at finite chemical potential due to a notorious sign problem. Perturbative calculations are only useful at very high temperatures as the QCD coupling constant becomes small. In recent years, relativistic fluid dynamics and statistical mechanics emerged as two important theoretical tools to explore the properties of hot and dense QCD matter created in the heavy ion collision experiments. Standard statistical models or thermal models assume a system in global thermodynamical equilibrium. It means that the intensive variables like pressure $(P)$, temperature $(T)$, and chemical potential $(\mu)$ are constant throughout the volume of the system. The most popular thermal model is ideal hadron gas (IHG) model and its modified versions are known as excluded volume models. These models assume that the hadrons are produced according to their statistical weights from an equilibrated hadron gas at freeze-out. These models were used in the recent past in order to deduce the multiplicities and ratios of hadrons, and agreements with the experimental data were found to be excellent [18-21]. On the other hand, hydrodyanmical or transport models assume that the system created at initial time is in local thermodynamic equilibrium $[22,23]$. It means that the thermodynamical variables such as pressure and temperature of the system vary with space-time point. However, the variation is so slow that, for any point, one can assume thermodynamic equilibrium in some neighborhood about that point. After this initial time, the system expands and cools off until the hadronic freeze-out occurs and produces the hadrons. These models again show good agreement with the data obtained from RHIC and LHC [23].

In this paper, our main motivation is to show what one should expect in context to particle multiplicities and particle ratios at CBM energies, in view of equilibrated statistical as well as transport models. We further compare the model results with the available experimental data of AGS and SPS energies. The rest of the paper is organized as follows: in the second section, we will provide a brief description of a recently developed thermal model with excluded volume correction. Further Section 3 will provide the results regarding the hadron multiplicities and their ratios and comparison among various models. We mainly emphasize the net baryon density created at freeze-out, the production of strange particles and the asymmetry in the particle-antiparticle production, and so forth because these issues are still unsettled in order to get information on the QCD phase transition. The last section will be devoted to the conclusion and future prospects drawn from this study.

\section{EOS for a Hot and/or Dense Hadron Gas}

Recently we have proposed [24-28] a thermodynamically consistent excluded volume model for the hot and dense hadron gas (HG). In this model, the grand canonical partition function for the HG with full quantum statistics and after suitably incorporating excluded volume correction is [24-28]

$$
\ln Z_{i}^{\mathrm{ex}}=\frac{g_{i}}{6 \pi^{2} T} \int_{V_{i}^{0}}^{V-\sum_{j} N_{j} V_{j}^{0}} d V
$$

$$
\times \int_{0}^{\infty} \frac{k^{4} d k}{\sqrt{k^{2}+m_{i}^{2}}} \frac{1}{\left[\exp \left(\left(E_{i}-\mu_{i}\right) / T\right)+1\right]},
$$

where $g_{i}$ is the degeneracy factor of $i$ th species of baryons, $E_{i}$ is the energy of the particle $\left(E_{i}=\sqrt{k^{2}+m_{i}^{2}}\right), V_{i}^{0}$ is the eigenvolume of one baryon of $i$ th species and $\sum_{j} N_{j} V_{j}^{0}$ is the total occupied volume, and $N_{j}$ represents total number of baryons of $j$ th species.

Now we can write (1) as follows:

$$
\ln Z_{i}^{\mathrm{ex}}=V\left(1-\sum_{j} n_{j}^{\mathrm{ex}} V_{j}^{0}\right) I_{i} \lambda_{i},
$$

where $I_{i}$ represents the integral:

$$
I_{i}=\frac{g_{i}}{6 \pi^{2} T} \int_{0}^{\infty} \frac{k^{4} d k}{\sqrt{k^{2}+m_{i}^{2}}} \frac{1}{\left[\exp \left(E_{i} / T\right)+\lambda_{i}\right]},
$$

and $\lambda_{i}=\exp \left(\mu_{i} / T\right)$ is the fugacity of the particle, $n_{j}^{\text {ex }}$ is the number density of $j$ th type of baryons after excluded volume correction, and it can be obtained from (2) as follows:

$$
n_{i}^{\mathrm{ex}}=\frac{\lambda_{i}}{V}\left(\frac{\partial \ln Z_{i}^{\mathrm{ex}}}{\partial \lambda_{i}}\right)_{T, V} .
$$

This leads to a transcendental equation:

$$
n_{i}^{\mathrm{ex}}=(1-R) I_{i} \lambda_{i}-I_{i} \lambda_{i}^{2} \frac{\partial R}{\partial \lambda_{i}}+\lambda_{i}^{2}(1-R) I_{i}^{\prime},
$$

where $I_{i}^{\prime}$ is the partial derivative of $I_{i}$ with respect to $\lambda_{i}$ and $R=\sum_{i} n_{i}^{\mathrm{ex}} V_{i}^{0}$ is the fractional occupied volume depending on $n_{i}^{\mathrm{ex}}$. We can write $R$ in an operator equation as follows [24]:

$$
R=R_{1}+\widehat{\Omega} R,
$$

where $R_{1}=R^{0} /\left(1+R^{0}\right)$ with $R^{0}=\sum n_{i}^{0} V_{i}^{0}+\sum I_{i}^{\prime} V_{i}^{0} \lambda_{i}^{2} ; n_{i}^{0}$ is the density of pointlike baryons of $i$ th species and the operator $\widehat{\Omega}$ has the following form:

$$
\widehat{\Omega}=-\frac{1}{1+R^{0}} \sum_{i} n_{i}^{0} V_{i}^{0} \lambda_{i} \frac{\partial}{\partial \lambda_{i}} .
$$

Using Neumann iteration method and retaining the series up to $\widehat{\Omega}^{2}$ term, we get

$$
R=R_{1}+\widehat{\Omega} R_{1}+\widehat{\Omega}^{2} R_{1} .
$$

Equation (8) can be solved numerically. Finally, we get the total pressure $[24,25]$ of the hadron gas as follows:

$$
P_{\mathrm{HG}}^{\mathrm{ex}}=T(1-R) \sum_{i} I_{i} \lambda_{i}+\sum_{i} P_{i}^{\mathrm{meson}} .
$$

In (9), the first term represents the pressure due to all types of baryons, where excluded volume correction is 
incorporated and the second term gives the total pressure due to all mesons in HG having a pointlike size. This makes it clear that we consider the hard-core repulsion arising between two baryons only. Essentially, we consider that the mesons can interpenetrate each other but baryons can not owing to their hard-core size. In this calculation, we have taken an equal volume $V^{0}=4 \pi r^{3} / 3$ for each type of baryon with a hardcore radius $r=0.8 \mathrm{fm}$. We have taken all baryons and mesons and their resonances having masses up to $2 \mathrm{GeV} / c^{2}$ in our calculation for the HG pressure. We have also imposed the condition of strangeness conservation by putting $\sum_{i} S_{i}\left(n_{i}^{s}-\right.$ $\left.\bar{n}_{i}^{s}\right)=0$, where $S_{i}$ is the strangeness quantum number of the $i$ th hadron and $n_{i}^{s}\left(\bar{n}_{i}^{s}\right)$ is the strange (antistrange) hadron density, respectively. Using this constraint equation, we get the value of strange chemical potential in terms of $\mu_{B}$.

In relativistic heavy ion collisions, a hot, dense matter is formed and it is often referred to as a "fireball." The physical variables of the fireball are the volume $V$, energy density $\epsilon$, and baryon density $\rho_{B}$, which are in fact related to $T$ and $\mu_{B}$ of the fireball. When cooling or expansion of the fireball starts, it goes through two types of freeze-out stages, namely, chemical freeze-out and thermal freeze-out. When inelastic collisions between constituents of the fireball cease, we call this the chemical freeze-out stage. Later when elastic collisions are also stopped, this is called the thermal freeze-out. In our model, we suppose that at the time of chemical freeze-out all the hadrons are emitted from the entire freeze-out hypersurface. Now the main point is to correlate thermodynamical variables $T$ and $\mu_{B}$ of the fireball to the experimental variable $\sqrt{s_{N N}}$. In our model, we vary the $T$ and $\mu_{B}$ of our model and observe how the experimental data of particle ratio are fitted at a given $\sqrt{s_{N N}}$. Similarly, we repeat the exercise for all the data available ranging from SIS to RHIC energy and thus get a set of $T$ and $\mu_{B}$ for a particular $\sqrt{s_{N N}}$. Now after such fitting procedure, we get a parameterization between $\sqrt{s_{N N}}$ and $\mu_{B}$ or $T$ as follows:

$$
\begin{aligned}
& \mu_{B}=\frac{a}{1+b \sqrt{s_{N N}}}, \\
& T=c-d \mu_{B}^{2}-e \mu_{B}^{4}
\end{aligned}
$$

where the parameters $a, b, c, d$, and $e$ have been determined from the best fit: $a=1.482 \pm 0.003 \mathrm{GeV}, b=0.351 \pm$ $0.009 \mathrm{GeV}^{-1}, c=0.163 \pm 0.002 \mathrm{GeV}, d=0.170 \pm 0.020 \mathrm{GeV}^{-1}$, and $e=0.015 \pm 0.010 \mathrm{GeV}^{-3}$. The details of the fitting procedure in thermal models can be found in [18]. We have also done this fitting for various other hadron gas models in order to obtain the values of $T$ and $\mu_{B}$ with respect to $\sqrt{s_{N N}}$ and all this is shown in Table I of [24].

\section{Results and Discussion}

The extracted freeze-out temperature in statistical thermal models of HG generally increases monotonically with the collision energy. However, the corresponding net baryon density exhibits a more complicated behavior [36]. Figure 1 demonstrates the variation of $\rho_{B}$ with $\sqrt{s_{N N}}$. To calculate $\rho_{B}$, we first use our parameterization given by (10) to get the

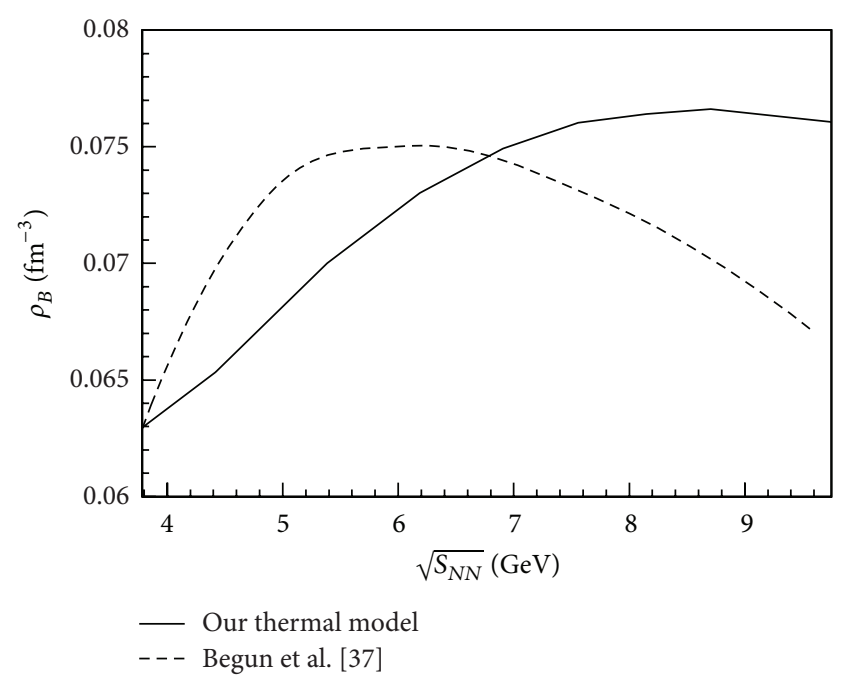

FIGURE 1: Variation of net baryon density $\left(\rho_{B}\right)$ at freeze-out with respect to center-of-mass energy $\left(\sqrt{s_{N N}}\right)$.

values of $\mu_{B}$ and $T$ for given $\sqrt{s_{N N}}$. At these $T$ and $\mu_{B}$, we have calculated the value of net baryon density $\rho_{B}$. In the present excluded volume model, the net baryon density increases with $\sqrt{s_{N N}}$ (see Figure 1), reaches a maximum value near $\sqrt{s_{N N}}=8-9 \mathrm{GeV}$, and then starts decreasing. The maximum freeze-out net baryon density is approximately half of the normal nuclear density, $\rho_{0}=0.15 \mathrm{fm}^{-3}$. We have compared our model results with the net baryon density obtained by Begun et al. [37] using excluded volume model based on [38]. They have calculated the baryon density at various $\sqrt{s_{N N}}$ using $0.5 \mathrm{fm}$ as hard-core radius $\left(r_{b}\right)$ for each baryon and all the mesons are treated as pointlike particles, that is, $\left(r_{m}=0\right)$. On the other hand, we have taken the value of $0.8 \mathrm{fm}$ as the hard-core radius for each baryon. The quantitative values of maximum freeze-out density achieved in heavy ion collisions are almost equal in both models. However, the position of the maximum in the net baryon density is somewhat shifted towards the lower energy side in the results obtained by Begun et al. [37]. The maximum value of $\rho_{B}$ obtained in our model as well as in the excluded volume model of Begun et al. is also lower than the value obtained in [36] where an HRG model is used without any excluded volume correction. Obviously, the excluded volume effect shifts the net baryon density achieved at freeze-out towards the lower value.

Figure 2 presents the variation of total multiplicity of $\pi^{+}$ with respect to $\sqrt{s_{N N}}$. In the CBM energy range, the fireball volume at freeze-out, extracted in the excluded volume model approach, appears almost constant [28] for all emitted particles. We have taken $5000 \mathrm{fm}^{3}$ as the fireball volume in order to calculate the total multiplicity of hadrons. We compare our results with the experimental data obtained at AGS and SPS [30-35] at low energies. We have also shown the total multiplicity of $\pi^{+}$obtained from transport models like HSD and UrQMD. Both HSD [29] and UrQMD [39, 40] models usually employ the concepts of string, quark, and diquark, $(q, \bar{q}, q q, \bar{q} \bar{q})$ as well as the hadronic degrees of freedom. However, the numerical evaluations are quite different in 


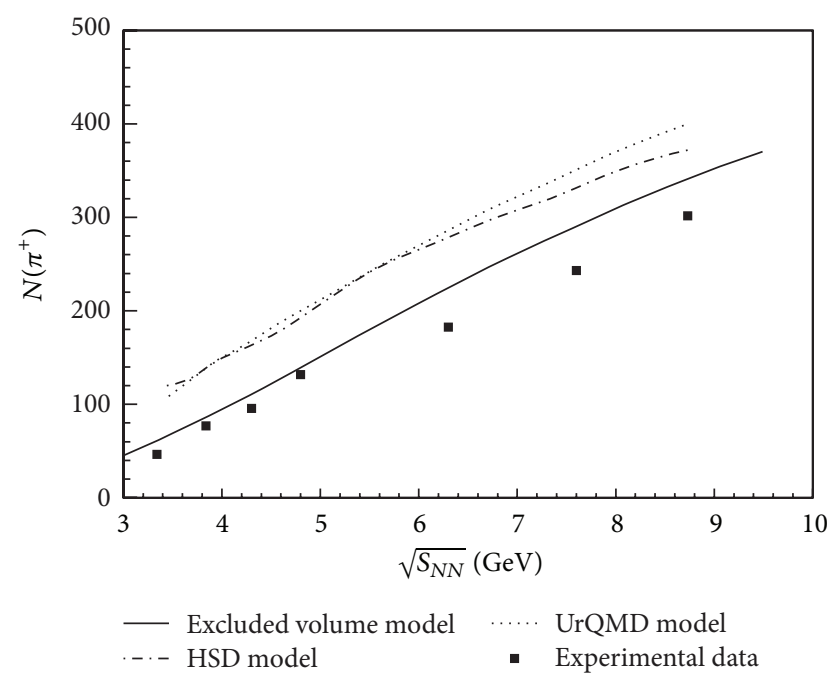

FIGURE 2: Variation of total multiplicity of produced $\pi^{+}$with respect to $\sqrt{s_{N N}}$. Dash-dotted and dotted curves are the results obtained from HSD and UrQMD models, respectively [29]. Experimental data is taken from [30-35].

HSD as compared to UrQMD. The UrQMD includes all baryonic resonances up to an invariant mass of $2 \mathrm{GeV}$ as well as mesonic resonances up to $1.9 \mathrm{GeV}[39,40]$. However, HSD incorporates only the baryon octet and decuplet states and $N^{*}(1440), N^{*}(1535)$ as well as their antiparticles together with the $0^{-}$and $1^{-}$meson octets. Higher baryonic resonances are discarded as the resonance structure (above $\Delta$ peak) is not clearly seen experimentally even in the photoabsorption by light nuclei [41-43]. In contrast to the UrQMD at low energy baryon-baryon and meson-baryon collisions, HSD includes the direct (nonresonant) meson production. Our excluded volume model suitably describes the data up to $5 \mathrm{GeV}$. However, all three models yield larger multiplicity for $\pi^{+}$relative to the data above $5 \mathrm{GeV}$, although the multiplicity of the produced $\pi^{+}$in excluded volume model is closer to the data in comparison to other two models. In principle, the yield of each particle is mainly governed by the particle fugacity essentially determined from the chemical freeze-out parameters. It also depends on the size of the system (or volume $V$ ) in which some variations can always be thought to appear.

Figure 3 demonstrates the variation of $\mathrm{K}^{+}$multiplicity with respect to $\sqrt{s_{N N}}$. We have used the same freeze-out volume, that is, $5000 \mathrm{fm}^{3}$, as that used in the $\pi^{+}$multiplicity. We compare our model results with the results obtained from HSD and UrQMD simulations. We have also shown the experimental data from CERN-SPS and RHIC-AGS for comparison [30-35]. The results obtained from both HSD and UrQMD do not match with the data beyond the energy $5 \mathrm{GeV}$. The overall level of agreement with the excluded volume model results is quite good. However, HSD results also suitably describe the data below $5 \mathrm{GeV}$. It should be emphasized here that the thermal statistical model should be used with caution at lower energies since the number of produced particles is very small.

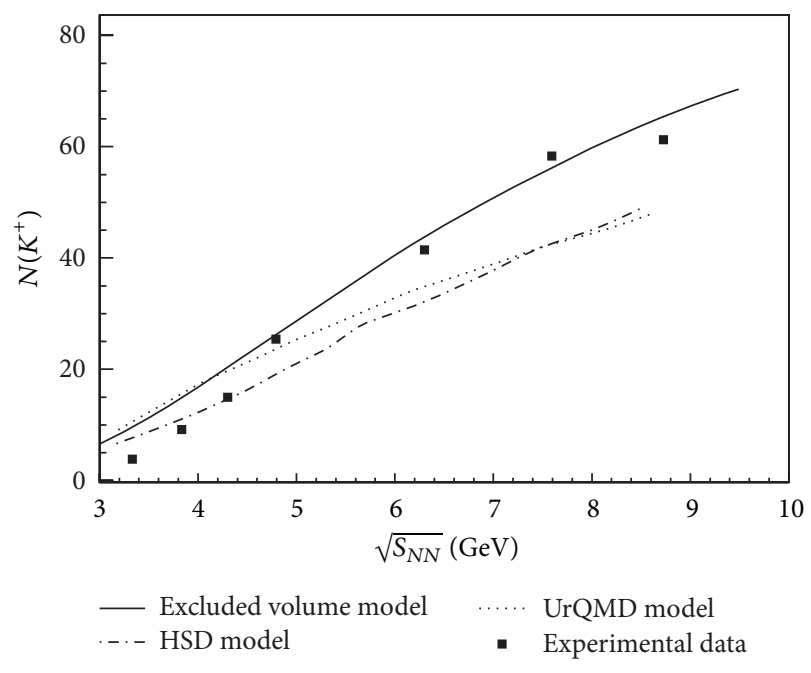

FIGURE 3: Variation of total multiplicity of produced $\mathrm{K}^{+}$with respect to $\sqrt{s_{N N}}$. Dash-dotted and dotted curves are the results obtained from HSD and UrQMD models, respectively [29]. Experimental data is taken from [30-35].

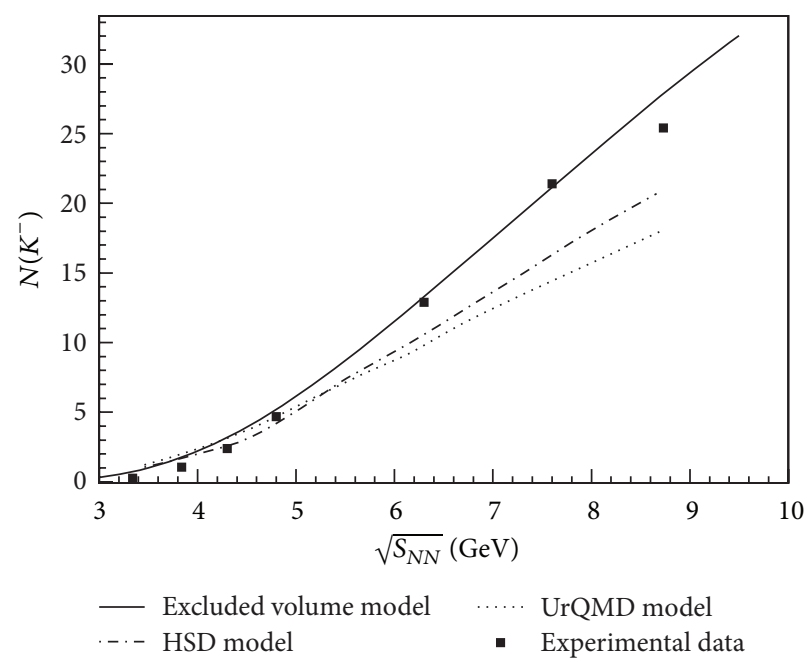

FIGURE 4: Variation of total multiplicity of produced $K^{-}$with respect to $\sqrt{s_{N N}}$. Dash-dotted and dotted curves are the results obtained from HSD and UrQMD models, respectively [29]. Experimental data is taken from [30-35].

In Figure 4, we present the variation of total multiplicity of $K^{-}$with $\sqrt{s_{N N}}$. The results obtained from our model are in excellent agreement with the data [30-35]. However, HSD and UrQMD results satisfy the experimental data only below $5 \mathrm{GeV}$. Above $5 \mathrm{GeV}$, the total multiplicity of $K^{-}$obtained from HSD and UrQMD is relatively small and does not agree with the data.

Strangeness enhancement has been proposed as one of the early and important signals of QGP formation [45-49]. It has been argued that if a quark-gluon plasma is formed from compressed nuclear matter as it may happen in the nuclear fragmentation region and/or in the low energy "stopping 
regime," then the abundance of $s$ and $\bar{s}$ quark would be highly enhanced compared to that of light $u$ or $d$ quark $[45,46,50,51]$. This is possibly due to the Pauli exclusion principle which strongly suppresses the further creation of light-quark pairs [52] in the medium having abundant $u, d$ quarks. This asymmetry in the flavor composition generated by a baryon-rich QGP should result in a large production of $K^{+}, K^{-}, \Lambda, \bar{\Lambda}$, and so forth [53]. This effect is even more evident in the case of the multistrange hyperons. A striking observation reported by NA49 collaboration is a pronounced and a sharp maximum in the excitation function of $K^{+} / \pi^{+}$ ratio at $30 \mathrm{AGeV}$ [31]. This sharp maximum which is also known as "horn" is not seen in $\mathrm{p}+\mathrm{p}$ collisions. As $\mathrm{K}^{+}$is by far the most abundant carrier of antistrangeness at SPS energies, it also provides a good measure of the total strangeness produced in the collision. The ratio $K^{+} / \pi^{+}$represents the strangeness to entropy ratio. A sharp maximum in this quantity was predicted by the statistical model indicating the early stage as a consequence of the transition to a deconfined state [53]. A similar maximum at the same beam energy is also reported by the same collaboration for other strange particles like $\Lambda$ 's and $\Xi^{-}$[32]. These observations confirm that this particular feature is not given by $\mathrm{K}^{+}$alone, but they represent the total strangeness content of the final state [54]. The measurement of the excitation function of strangeness production by NA49 collaboration has renewed a fresh stimulating discussion about the role of strangeness as a signature for the deconfinement phase transition.

Figure 5 demonstrates the variation of $\mathrm{K}^{+} / \pi^{+}$ratio with respect to $\sqrt{s_{N N}}$. We have compared our model results with the experimental data obtained from SPS experiment [30-35]. We have further compared them with the other results like HSD, UrQMD, and Boltzmann-UehlingUhlenbeck (BUU) transport [44] models. We find that both HSD and UrQMD models fail to give agreement with the experimental data. However, BUU model shows better results in comparison to HSD and UrQMD. Our model yields better results in agreement with the experimental data. The authors in [29] have suggested that the overestimation of $\pi^{+}$multiplicity in thermal models gives theoretical curve lying below the experimental data. The similar fireball volume used for $\mathrm{K}^{+}$and $\pi^{+}$in the excluded volume model can also contribute to such disagreement. Here, we use the same freeze-out volume for the emission of all kinds of hadrons from the fireball surface. CBM experiment will definitely provide an important insight in the understanding of strange particle production mechanism and more vitally address the mechanism for the existence of the "horn" like behavior in the lower energy region.

In Figure 6 , we present the variation of $K^{-} / \pi^{-}$with respect to $\sqrt{s_{N N}}$. Our model suitably describes the data, but below $5 \mathrm{GeV}$ we again notice a large disagreement. However, HSD and UrQMD both also give complete disagreement with the experimental data almost in the entire energy range, that is, from 3 to $9 \mathrm{GeV}$.

A large production of anti-baryons over baryons in heavy ion collisions are considered as the signal of QGP formation [55-59]. In heavy ion collisions, the system has a nonzero

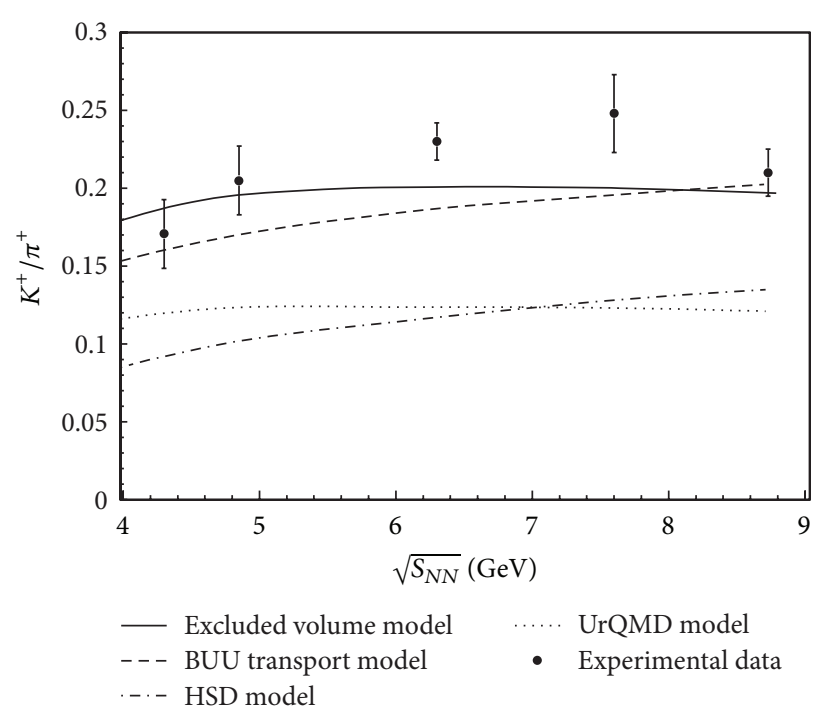

FIGURE 5: Variation of $K^{+} / \pi^{+}$ratio with respect to $\sqrt{s_{N N}}$. The results obtained from HSD and UrQMD are taken from [29]. The results obtained from BUU transport model are extracted from [44]. Experimental data is taken from [30-35].

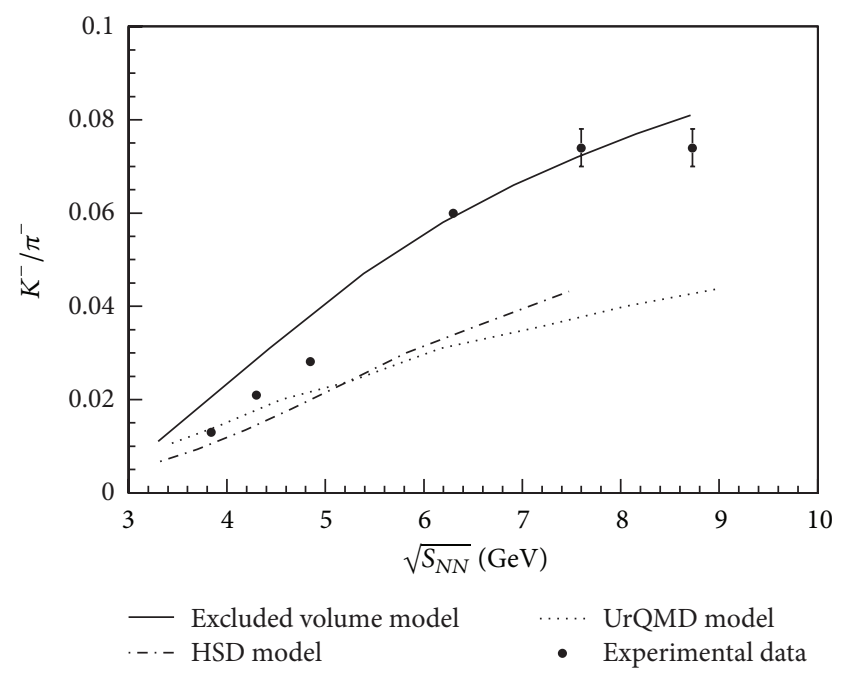

FIGURE 6: Variation of $K^{-} / \pi^{-}$ratio with respect to $\sqrt{s_{N N}}$. The results obtained from HSD and UrQMD are taken from [29]. Experimental data is taken from [30-35].

baryon number density arising due to nuclear stopping. At small and moderate center-of-mass energies as existing in the case of CBM experiment, the nuclear stopping is large in comparison to the RHIC and/or LHC energies where nuclear transparency is found to dominate. Nuclear stopping leads to an asymmetry between the production of hadrons and antihadrons in the produced particles other than the initial finite baryon number. However, there is a possibility that hadronization can also generate additional particleantiparticle asymmetry. This asymmetry can be measured by the ratio of yields of antihadrons to hadrons $[60,61]$. Ratios of yield of antiprotons to protons $(\bar{p} / p)$ and that of antikaons to 


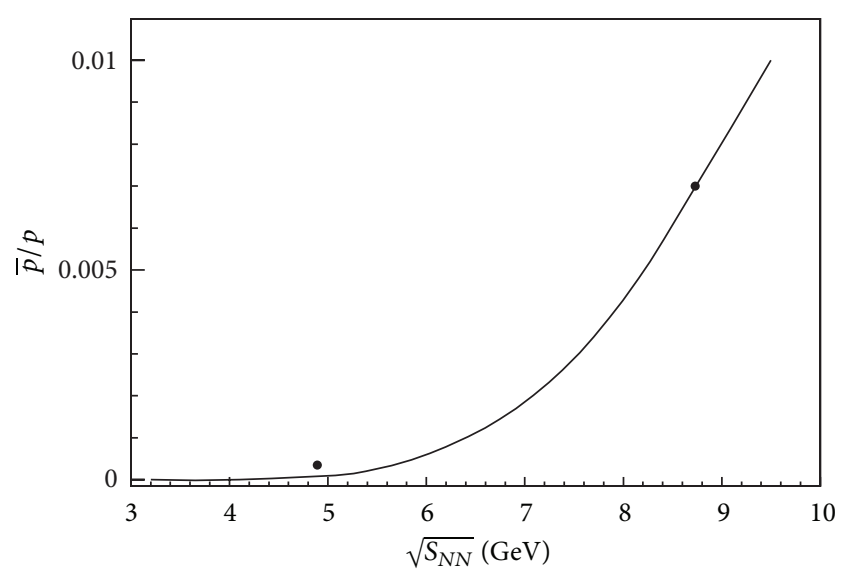

Figure 7: Variation of $\bar{p} / p$ with respect to $\sqrt{s_{N N}}$. Experimental data is taken from [30-35].

kaons $\left(K^{-} / K^{+}\right)$are the representatives of two such significant observables measuring the hadron-antihadron asymmetry in heavy ion collisions $[60,61]$. The ratio $\bar{p} / p$ carries the information regarding baryon-antibaryon asymmetry and the ratio $K^{-} / K^{+}$almost cancels the effect of strangeness production and indicates the asymmetry between charged mesons and their antiparticles generated in the hot, dense medium.

In Figures 7 and 8, we have shown the variations of $\bar{p} / p$ and $K^{-} / K^{+}$with $\sqrt{s_{N N}}$, respectively, as obtained in our excluded volume model with the center-of-mass energy. In both cases, the production of antiparticle to particle is less at lowest CBM energy. However, the asymmetry in $\bar{p}$ and $p$ production is larger as also observed between $K^{-}$and $K^{+}$. As the energy increases, the production of antiparticle over particle increases since there is an increase in nuclear transparency. However, it is important to state here that the excluded volume model does not agree with the experimental data for the net nucleon density at RHIC highest energy [24] also.

In summary, we have calculated the net baryon density at freeze-out in the CBM energy range which comes out to be the maximum achievable density in heavy ion collisions. Further, we have calculated the total multiplicities of various produced hadrons, for example, $\pi^{+}, K^{+}, K^{-}$using a constant freeze-out volume which is equal to $5000 \mathrm{fm}^{3}$ for all the hadrons. We have also calculated the ratio of $K^{+} / \pi^{+}$and $K^{-} / \pi^{-}$in CBM energy range. Almost all the models fail to reproduce the "horn" in $\mathrm{K}^{+} / \pi^{+}$ratio. Furthermore, we have also calculated the particle to antiparticle ratio like $\bar{p} / p$ and $K^{-} / K^{+}$. In CBM energy range, these ratios increase rapidly. However, due to lack of experimental data, we do not have any meaningful comparison, and hence we cannot precisely understand the asymmetry between hadron and antihadron production in heavy ion collisions.

In conclusion, we face many worthwhile questions regarding particle production at CBM energy regime. More vital questions are regarding the formation of a thermal

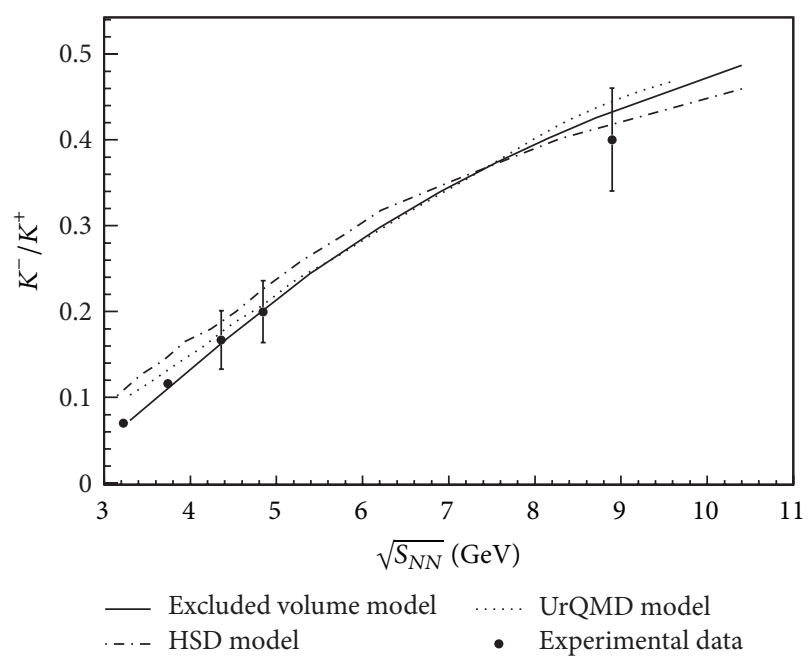

FIGURE 8: Variation of $K^{-} / K^{+}$with respect to $\sqrt{s_{N N}}$. The results obtained from HSD and UrQMD are taken from [29]. Experimental data is taken from [30-35].

fireball and the applicability of thermal equilibrium in the statistical system. But it is interesting to investigate what we should precisely expect. So we should precisely know what we expect from various equlibrium-nonequilibrium models. Our excluded volume model gives a better agreement with the available experimental data in comparison to other models. In the CBM energy range, most of the multiplicities as well as particle ratios show somewhat peculiar behavior. However, there is scarcity of experimental data. The data available suffer from poor statistics also. The upcoming CBM experiment having a high luminosity beam will provide a unique opportunity to perform systematic and comprehensive measurements, with better statistics of bulk and rare particle behavior. This will help us to understand the particle production mechanism and also possibly find the existence of QGP at CBM energy.

\section{Conflict of Interests}

The authors declare that there is no conflict of interests regarding the publication of this paper.

\section{Acknowledgments}

The authors gratefully acknowledge Professor C. P. Singh for many stimulating and inspiring discussions on the paper. One of the authors, P. K. Srivastava, is grateful to the University Grants Commission (UGC), New Delhi, for providing a research fellowship. A. Prakash and B. K. Singh are grateful to the Department of Science and Technology (DST), Government of India, New Delhi, for providing a research grant.

\section{References}

[1] C. P. Singh, "Signals of quark-gluon plasma," Physics Reports, vol. 236, no. 3, pp. 147-224, 1993. 
[2] C. P. Singh, "Current status of properties and signals of quarkgluon plasma," International Journal of Modern Physics A, vol. 7, no. 29, p. 7185, 1992.

[3] H. Satz, "quark matter and nuclear collisions: a brief history of strong interaction thermodynamics," International Journal of Modern Physics E, vol. 21, Article ID 1230006, 23 pages, 2012.

[4] B. Friman, C. Hohne, J. Knoll et al., Eds., The CBM Physics Book, vol. 814 of Lecture Notes in Physics, Springer, Berlin, Germany, 2011.

[5] A. Kumar, P. K. Srivastava, B. K. Singh, and C. P. Singh, "Charged hadron multiplicity distribution at relativistic heavy ion colliders," Advances in High Energy Physics, vol. 2013, Article ID 352180, 27 pages, 2013.

[6] A. Kumar, B. K. Singh, P. K. Srivastava, and C. P. Singh, "Wounded quarks and multiplicity atrelativistic ion colliders," The European Physical Journal Plus, vol. 128, p. 45, 2013.

[7] B. Mohanty, "STAR experiment results from the beam energy scan program at the RHIC," Journal of Physics G, vol. 38, no. 12, Article ID 124023, 2011.

[8] L. Adamczyk, J. K. Adkins, G. Agakishiev et al., "Observation of an energy-dependent difference in elliptic flow between particles and anti-particles in relativistic heavy ion collisions," Physical Review Letters, vol. 110, no. 14, Article ID 142301, 7 pages, 2013.

[9] B. B. Back, M. D. Bakerb, M. Ballintijn et al., "The PHOBOS perspective on discoveries at RHIC," Nuclear Physics A, vol. 757, no. 1-2, pp. 28-101, 2005.

[10] A. Laszlo, PoS(CPOD07), 054, 2007.

[11] F. Antinori, P. A. Bacon, A. Badalà et al., "Strangeness enhancements at central rapidity in $40 \mathrm{~A} \mathrm{GeV} / \mathrm{c} \mathrm{Pb}-\mathrm{Pb}$ collisions," Journal of Physics G, vol. 37, no. 4, Article ID 045105, 2010.

[12] R. Singh, L. Kumar, P. K. Netrakanti, and B. Mohanty, "Selected experimental results from heavy-ion collisions at LHC," Advances in High Energy Physics, vol. 2013, Article ID 761474, 22 pages, 2013.

[13] P. Senger, "The compressed baryonic matter experiment at FAIR,” Central European Journal of Physics, vol. 10, no. 6, pp. 1289-1294, 2012.

[14] P. Senger, "Probing dense baryonic matter," Progress in Particle and Nuclear Physics, vol. 62, no. 2, pp. 375-380, 2009.

[15] S. Borsanyi, G. Endrodi, Z. Fodor et al., "The QCD equation of state with dynamical quarks," Journal of High Energy Physics, vol. 2010, no. 11, article 77, 2010.

[16] S. Borsanyi, Z. Fodor, S. D. Katz et al., "Fluctuations of conserved charges at finite temperature from lattice QCD," Journal of High Energy Physics, vol. 2012, no. 1, article 138, 2012.

[17] S. Borsanyi, Z. Fodor, S. D. Katz et al., "Correlations and fluctuations from lattice QCD," Journal of Physics G, vol. 38, no. 12, Article ID 124060, 2011.

[18] A. Andronic, P. Braun-Munzinger, and J. Stachel, "Hadron production in central nucleus-nucleus collisions at chemical freeze-out," Nuclear Physics A, vol. 772, no. 3-4, pp. 167-199, 2006.

[19] J. Cleymans, H. Oeschler, and K. Redlich, "Statistical model description of $K^{+}$and $K^{-}$production between $1-10 A \cdot G e V$," Physics Letters B, vol. 485, pp. 27-31, 2000.

[20] F. Becattini, J. Cleymans, A. Keranen, E. Suhonen, and K. Redlich, "Features of particle multiplicities and strangeness production in central heavy ion collisions between 1.7A and 15A GeV/c," Physical Review C, vol. 64, no. 2, Article ID 024901, 9 pages, 2001.
[21] W. Broniowski and W. Florkowski, "Description of strange particle production in $\mathrm{Au}+\mathrm{Au}$ collisions of $\sqrt{s_{N N}}=130 \mathrm{GeV}$ in a single-freeze-out model," Physical Review C, vol. 65, no. 6, Article ID 064905, 6 pages, 2002.

[22] J. Y. Ollitrault, "Relativistic hydrodynamics for heavy-ion collisions," European Journal of Physics, vol. 29, no. 2, p. 275, 2008.

[23] U. Heinz and R. Snellings, "Collective flow and viscosity in relativistic heavy-ion collisions," Annual Review of Nuclear and Particle Science, vol. 63, pp. 123-151, 2013.

[24] S. K. Tiwari, P. K. Srivastava, and C. P. Singh, "Description of hot and dense hadron-gas properties in a new excluded-volume model," Physical Review C, vol. 85, no. 1, Article ID 014908, 15 pages, 2012.

[25] P. K. Srivastava and C. P. Singh, "Hybrid model for QCD deconfining phase boundary," Physical Review D, vol. 85, no. 11, Article ID 114016, 10 pages, 2012.

[26] P. K. Srivastava and C. P. Singh, "Strongly interacting matter at finite chemical potential: hybrid model approach," International Journal of Modern Physics A, vol. 28, no. 14, Article ID 1350051, 2013.

[27] S. K. Tiwari and C. P. Singh, "Particle production in ultrarelativistic heavy-ion collisions: a statistical-thermal model review," Advances in High Energy Physics, vol. 2013, Article ID 805413, 27 pages, 2013.

[28] S. K. Tiwari, P. K. Srivastava, and C. P. Singh, "The effect of flow on hadronic spectra in an excluded-volume model," Journal of Physics G, vol. 40, no. 4, Article ID 045102, 2013.

[29] H. Weber, E. L. Bratkovskaya, W. Cassing, and H. Stocker, "Hadronic observables at relativistic energies: anything strange with strangeness?" Physical Review C, vol. 67, no. 1, Article ID 014904, 19 pages, 2003.

[30] A. Andronic, D. Blaschke, P. Braun-Munzingera et al., "Hadron production in ultra-relativistic nuclear collisions: quarkyonic matter and a triple point in the phase diagram of QCD," Nuclear Physics A, vol. 837, no. 1-2, pp. 65-86, 2010.

[31] C. Alt, T. Anticic, B. Baatar et al., "Pion and kaon production in central $\mathrm{Pb}+\mathrm{Pb}$ collisions at $20 \mathrm{~A}$ and $30 \mathrm{~A} \mathrm{GeV}$ : evidence for the onset of deconfinement," Physical Review C, vol. 77, no. 2, Article ID 024903, 10 pages, 2008.

[32] C. Alt, T. Anticic, B. Baatar et al., "Energy dependence of $\Lambda$ and $\Xi$ production in central $\mathrm{Pb}+\mathrm{Pb}$ collisions at $20 \mathrm{~A}, 30 \mathrm{~A}$, $40 \mathrm{~A}, 80 \mathrm{~A}$, and $158 \mathrm{~A} \mathrm{GeV}$ measured at the CERN Super Proton Synchrotron," Physical Review C, vol. 78, no. 3, Article ID 034918, 15 pages, 2008.

[33] C. Blume, "Review of results from the NA49 Collaboration," Journal of Physics G, vol. 31, pp. S685-S691, 2005.

[34] S. V. Afanasiev, T. Anticic, D. Barna et al., "Energy dependence of pion and kaon production in central $\mathrm{Pb}+\mathrm{Pb}$ collisions," Physical Review C, vol. 66, no. 5, Article ID 054902, 9 pages, 2002.

[35] M. Gazdzicki, "Report from NA49," Journal of Physics G, vol. 30, no. 8, p. S701, 2004

[36] J. Randrup and J. Cleymans, "Maximum freeze-out baryon density in nuclear collisions," Physical Review C, vol. 74, no. 4, Article ID 047901, 3 pages, 2006.

[37] V. V. Begun, M. Gazdzicki, and M. I. Gorenstein, "Hadronresonance gas at freeze-out: reminder on the importance of repulsive interactions," Physical Review C, vol. 88, no. 2, Article ID 024092, 7 pages, 2013.

[38] D. H. Rischke, M. I. Gorenstein, H. Stöcker, and W. Greiner, "Excluded volume effect for the nuclear matter equation of state," Zeitschrift für Physik C, vol. 51, no. 3, pp. 485-489, 1991. 
[39] S. Scherer, S. A. Bass, M. Bleicher et al., "Critical review of quark gluon signatures," Progress in Particle and Nuclear Physics, vol. 42, pp. 279-293, 1999.

[40] M. Bleicher, E. Zabrodin, C. Spieles et al., "Relativistic hadronhadron collisions in the ultra-relativistic quantum molecular dynamics model," Journal of Physics G, vol. 25, no. 9, p. 1859, 1999.

[41] N. Bianchi, V. Muccifora, A. Deppman et al., "Absolute measurement of the total photoabsorption cross section for carbon in the nucleon resonance region," Physics Letters B, vol. 309, no. 1-2, pp. 5-9, 1993.

[42] N. Bianchi, V. Muccifora, E. de Sanctis et al., "Absolute total photoabsorption cross sections on nuclei in the nucleon resonance region," Physics Letters B, vol. 325, no. 3-4, pp. 333-336, 1994.

[43] N. Bianchi, V. Muccifora, E. de Sanctis et al., "Total hadronic photoabsorption cross section on nuclei in the nucleon resonance region," Physical Review C, vol. 54, no. 4, pp. 1688-1699, 1996.

[44] M. Wagner, A. B. Larionov, and U. Mosel, "Kaon and pion production in relativistic heavy-ion collisions," Physical Review C, vol. 71, no. 3, Article ID 034910, 19 pages, 2005.

[45] J. Rafelski and B. Muller, "Strangeness production in the quarkgluon plasma," Physical Review Letters, vol. 48, no. 16, pp. 10661069, 1982.

[46] J. Rafelski, "Strangeness production in the quark gluon plasma," Nuclear Physics A, vol. 418, pp. 215-235, 1984.

[47] C. P. Singh and S. Uddin, "Strangeness abundance as a signal for quark-gluon plasma," Physical Review D, vol. 41, no. 3, pp. 870-874, 1990.

[48] V. K. Tiwari and C. P. Singh, "Strangeness enhancement-a potential signature for QGP phase," Physics Letters B, vol. 411, no. 1-2, pp. 225-229, 1997.

[49] C. P. Singh, "Comment on ' $\varphi$-meson production as a probe of the quark-gluon plasma,' Physical Review Letters, vol. 56, no. 16, p. $1750,1986$.

[50] T. S. Biro and J. Zimanyi, "Quarkochemistry in relativistic heavy-ion collisions," Physics Letters B, vol. 113, pp. 6-10, 1982.

[51] T. S. Biro and J. Zimanyi, "Quark-gluon plasma formation in heavy ion collisions and quarkochemistry," Nuclear Physics A, vol. 395, pp. 525-538, 1983.

[52] S. Margetis, K. Safarik, and O. V. Baillie, "Strangeness production in heavy ion collisions," Annual Review of Nuclear and Particle Science, vol. 50, pp. 299-342, 2000.

[53] S. Uddin and C. P. Singh, "Dependence of $K / \pi$ ratio on temperature and chemical potential as a signature of quarkgluon plasma," Physics Letters B, vol. 278, no. 3, pp. 357-362, 1992.

[54] V. Friese, PoS(CPOD09), 005, 2009.

[55] U. Heinz, P. R. Subramanian, and W. Greiner, "Antinuclei-a signature for the quark-gluon plasma," Zeitschrift für Physik A, vol. 318, no. 2, pp. 247-248, 1984.

[56] P. Koch, B. Muller, H. Stocker, and W. Greiner, "Antibaryonbaryon ratios as a signal for quark gluon plasma for quark gluon plasma formation," Modern Physics Letters A, vol. 3, no. 8, p. 737, 1988.

[57] J. Ellis, U. Heinz, and H. Kowalski, "Anti-baryon production in heavy-ion collisions," Physics Letters B, vol. 233, no. 1-2, pp. 223230, 1989.

[58] S. Gavin, M. Gyulassy, M. Plumer, and R. Venugopalan, "Antiproton production as a baryonometer in ultrarelativistic heavy ion collisions," Physics Letters B, vol. 234, no. 1-2, pp. 175$179,1990$.

[59] H. Sorge, A. V. Keitz, R. Mattiello, H. Stocker, and W. Greiner, "String dynamics in hadronic matter," Zeitschrift für Physik C, vol. 47, no. 4, pp. 629-634, 1990.

[60] A. Tawfik, "Antiproton-to-proton ratios for ALICE heavy-ion collisions," Nuclear Physics A, vol. 859, no. 1, pp. 63-72, 2011.

[61] A. Tawfik, "Matter-antimatter asymmetry in heavy-ion collisions," International Journal of Theoretical Physics, vol. 51, no. 5, pp. 1396-1407, 2012. 

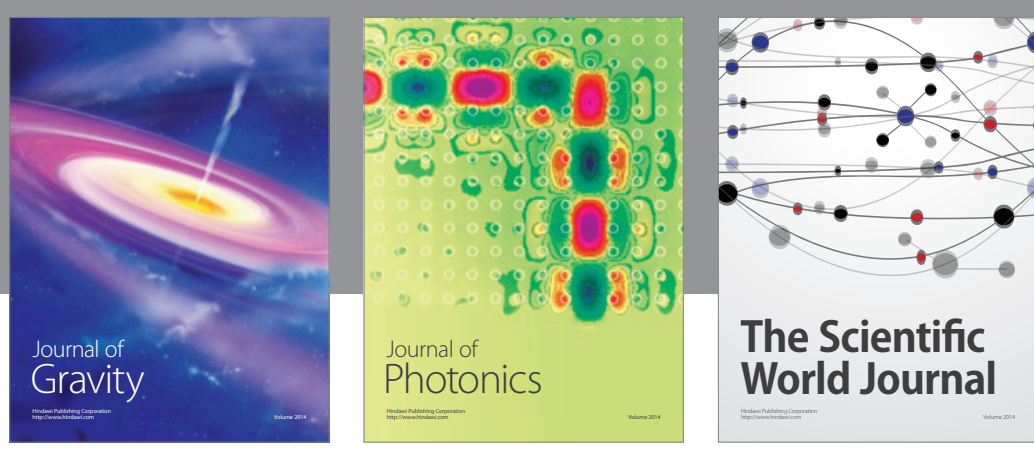

The Scientific World Journal
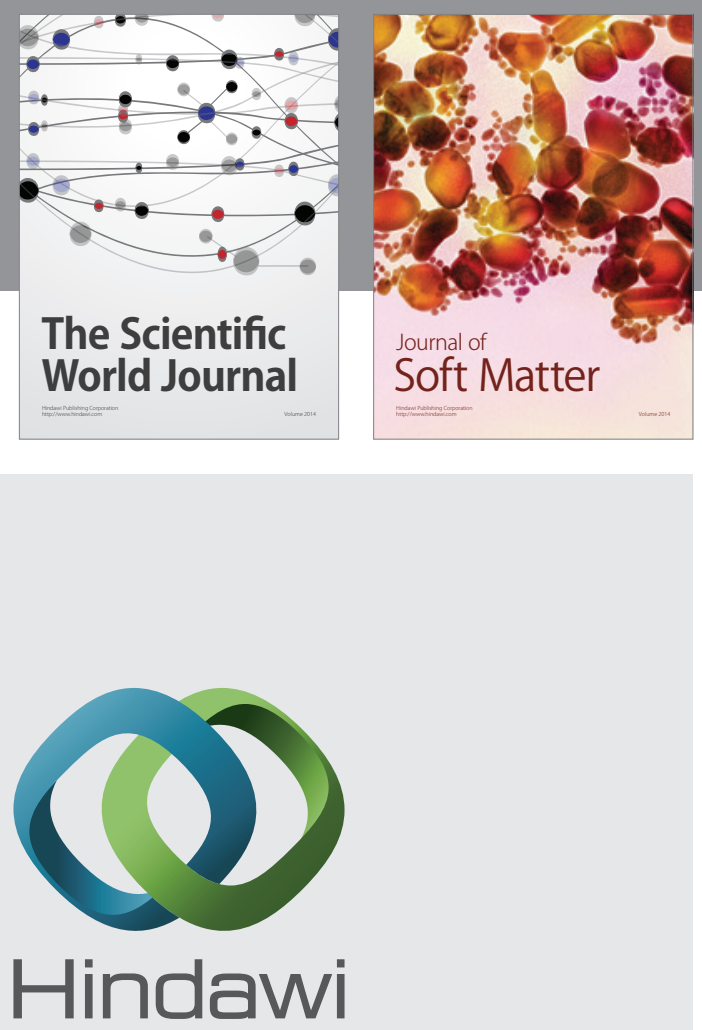

Submit your manuscripts at

http://www.hindawi.com

nternational Journal of

Statistical Mechanics

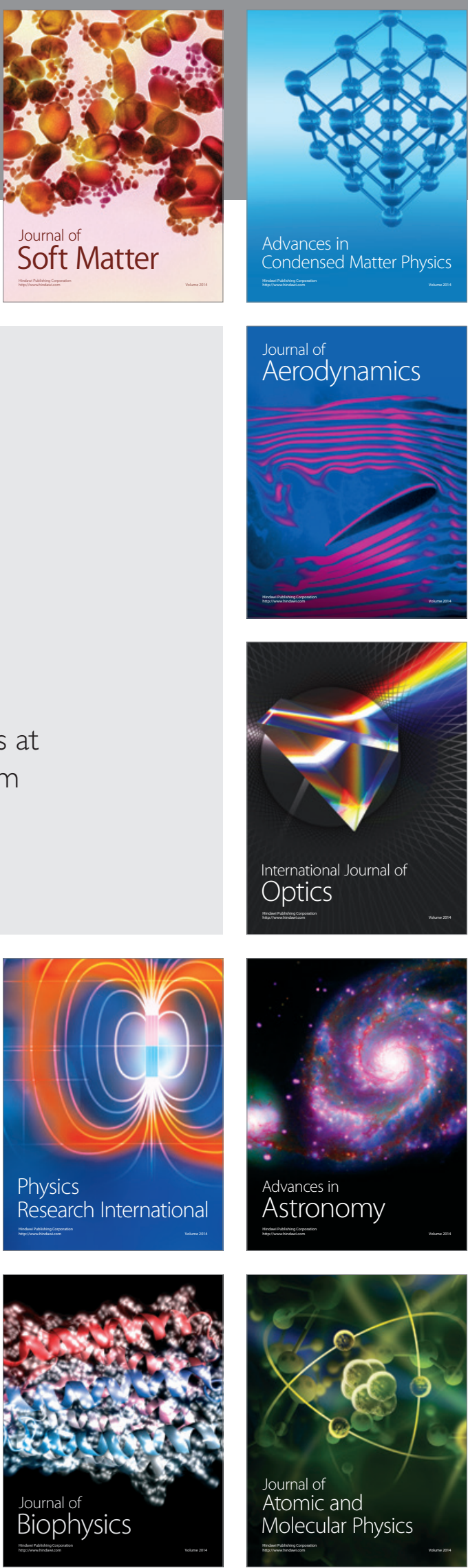